\title{
Reconstruction of Laryngotracheal War Injuries with the Median Layer of the Deep Cervical Fascia
}

\author{
D Danić \\ MD \\ D Miličić \\ MD \\ D Prgomet
}

MD

Dept of Otorhinolaryngology and Cervicofacial Surgery, General Hospital "Dr Josip Bencević" Slavonski Brod, Andrije Stampara 42, 55000 Slavonski Brod, Croatia.

\section{S Simovíc}

MD

Clinic of Otorhinolaryngology and Cervicofacial Surgery, Medical School Zagreb, Šalata 4, 41000 Zagreb, Croatia.

SUMMARY: Surgical exploration and immediate reconstruction with the median layer of the deep cervical fascial

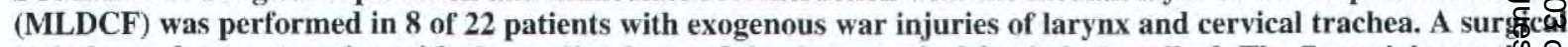
technique of reconstruction with the median layer of the deep cervical fascia is described. The 7 surviving patien? had good respiration without signs of stenosis of the larynx and/or the trachea. Four had good and 3 satisfactor $y$, phonation, and none had swallowing difficulties. Owing to the simplicity of the surgical approach, its size biological properties, the median layer of deep cervical fascia proved itself to be a suitable material in immediate reconstruction of exogenerous war injuries of the larynx and cervical trachea.

\section{Introduction}

Despite the superficial position of the larynx and trachea, the incidence of injury by exogenous factors is very rare during both peace-time and war (1). There were 13 patients treated at our department for exogenous injury of the larynx and the trachea during the period 1975 to 1984 , representing $0.94 \%$ of all hospitalized patients with head and neck injuries during that period (2).

Data on the incidence of combat related laryngotracheal injuries is rarely found in the literature. Zayton et al, recorded 54 such patients among 1,021 wounded with head and neck injuries during the ten-year Lebanon war (3). The data on the incidence of such injuries could vary greatly during the same war, for example in the II Mobile Surgical Hospital in South Vietnam, among 1,011 wounded there were only 6 tracheal and cricoid cartilage injuries in the year 1966 to 1967 (4). However, from 1967 to 1968,22 laryngotracheal injuries were registered in the "24th evacuation hospital in Vietnam" (5).

A number of authors have offered differing principles of treatment for peace-time injuries. These differing views and opinions are with regard to all stages of care; from indications, time of initial surgical care through to definitive surgical management (6-10). The absence of thyroid cartilage or exposure of tracheal cartilage surfaces, which favours infection and diminished functional results of reconstructive intervention, promided many authors to use different covering materials, 要角 varying success. Krajina has used fascia of the sternohyoid muscle in the reconstruction of the larygk lumen in various types of partial laryngectomy sinळe 1969, with excellent results (11). In the war situation made use of this technique, improving it and widening $\frac{\mathcal{Q}}{3} \mathrm{~s}$ indications. A flap formed from the median layer of the deep cervical fascia (MLDCF) being used in t immediate reconstruction of 8 cases of laryngotrache trauma.

\section{Patients and Methods}

During the 1991/1992 war in Croatia $(12,13), 7,0 \$_{3}$ wounded were treated at the Medical Center in Slavons Brod (the region of Brodska Posavina, East Croatia) froक्कु July 11991 to December 31 1992. Our department of the Otorhinolaryngology and Cervicofacial Surgery treatêd 728 head and neck injuries, of whom $22(0.31 \%)$ ha्रd laryngeal and cervical trachea injuries. Sixteen of the had an injury of the larynx, 4 of the larynx and trachea of the trachea and 1 of the recurrent laryngeal ner (Table 1).

Splinters of shells, mines and hand grenades caused injuries in 18, rifle bullet in 2 and plastic explosive in 1. A contusion was responsible for one other (Table 2). 
All wounded were male, 20 were soldiers and 2 were civilians, their ages ranging from 5 to 43 years. We hospitalised 21 patients, the patient with the laryngeal contusion being treated as an outpatient. Twenty wounded were hospitalised within the first three hours of injury. The patient with laryngeal contusion was treated on the second day after being wounded. The patient with the explosive laryngeal injury was transferred from another medical institution on the fifth day of the injury.

Only 2 of the wounded had isolated injuries of the larynx or trachea while the others had associated injuries of the neck and head or the neck and/or other parts of the body.

Endoscopic techniques, classical X-ray and CT-scan were used for pre-operative diagnosis.

The largest number of wounds were transglottic (nine), supraglottic (eight), glottic (three) and cricoid (six). Tracheal lesions were located in the cartilaginous skeleton up to the fourth tracheal ring and one of the wounded had a posterior membranous wall injury.

Twenty-one wounded were surgically treated, the one with the larynx contusion being treated conservatively. Eight of the 21 surgical patients were managed with median layer of the deep cervical fascia.

Surgically treated patients were divided into four groups (Table 3):

Table 1

War wounds of the head and neck treated in the General Hospital "Dr Josip Benč ević" Slavonski Brod from July 1 1991 to December 311992.

No. (\%) of patients/treatment

Outpatient Hospitalized Total

$\begin{array}{lrrrr}\text { Head } & 187(67) & 354(78) & 541(74) \\ \text { Neck } & 60(21) & 57(14) & 117(16) \\ \text { Head and neck } & 32(12) & 38(8) & 70(10) \\ \text { Total } & 279(38) & 449(62) & 728(100)\end{array}$

Table 2

The localization and etiology of war wounds of the larynx and trachea in 22 patients in General Hospital "Dr Josip

Bencevic" Slavonski Brod from July 11991 to December 31 1992.

\begin{tabular}{lrlr}
\hline Localization & (No.) & Etiology & (No.) \\
\hline Larynx & 16 & Shells & 18 \\
Trachea & 1 & Bullets & 2 \\
Larynx and trachea & 4 & Plastic explosive & 1 \\
Recurrent nerve & 1 & Contusion & 1
\end{tabular}

Table 3

Surgical treatment of 21 patients with laryngotracheal war

wounds.

\section{Group 1}

Hematomas, small lesions of laryngeal mucosa or cartilage

\section{Group 2}

Large lesions of mucosa especially at frontal junction, small fractures of the cartilage

\section{Group 3}

Large mucosal lesions, multiple fractures with defects of cartilage, preserved stability of cartilaginous skeleton

\section{Group 4}

Instability of the cartilaginous skeleton

\section{No. Treatment}

4 Surgical treatment of the wound without the explorationg of the larynx conservative debridement, primary suture of mucosa

10 Exploration of the larynx, MLDCF* reconstruction in 7 patients

3 Exploration of the larynx, MLDCF reconstruction in one patient, stent
4 Exploration of the larynx,

* MLDCF - median layer of deep cervical fascia used for reconstruction of laryngotracheal war injuries.

1. Four patients had hematomas, or small lesions endolaryngeal mucosa, and injury to the perichondritime but without fracture of the larynx or tracheal cartilagThese were treated surgically without exploration $\Phi$ the larynx or the trachea.

2. Four patients had minor lesions of the endolaryngena mucosa, especially in the area of the anterie commisure and smaller linear fractures of thyroid $\overrightarrow{\mathrm{k}} \mathrm{C}$ tracheal cartilage but without any large dislocation of ae defect. The lesions of perichondrium were not significant. These were treated by exploration, conservative debridement. The continuity of the mucosa was secured primarily by absorbable stitchesi 4- 0 or $5-0$.

3. Ten patients had large lesions of endolaryngeal mucosa with damage to vocal cords and/or ventricular folds, multiple fractures of thyroid and cricoid cartilages with defects in perichondrium and cartilage but with preserved stability of cricothyroid ring.

Seven patients were managed with use of the median? layer of the deep cervical fascia. In 5 a reconstruction of the laryngeal lumen was performed by use of this tissue. The sixth patient also had a partial defect of the first two tracheal rings so that the median layer of the deep cervical fascia was used for the reconstruction of the lumen of both the larynx and the trachea. The seventh patient, in addition to damage to the cricoidis and first tracheal ring, also had a penetrating wound oß the oesophagus and seventh cervical vertebra. In theo reconstruction of the cricoid and trachea a strap muscle flap, according to Miller-Duplechain (7), was used beside the median layer of the deep cervical fascia for
the prevention of tracheoesophageal fistula.

4. Three patients had massive lesions of the endolaryngea 5 structures, comminuted fractures and large defects oft 
thyroid and cricoid cartilage causing instability of the cricothyroid ring. The median layer of the deep cervical fascia was used for reconstruction in one patient.

\section{Surgical Technique}

The median layer of the deep cervical fascia consists of strong fibrous tissue encompassing only the anterior plane of the neck. It stretches between the omohyoid muscles and enclose the sternohyoid and sternothyroid muscles. Cranially it is tied to the hyoid bone and caudally to the posterior border of the sternum and clavicular incisure. Superficial and median fascia nearly merge in the medial line of the neck, but a thin layer of fat is preserved in the place where they are the closest (Fig. 1).

The median layer of the deep cervical fascia receives its vascular supply from branches of the superior and inferior thyroid arteries.

After tracheotomy and the formation of a tracheostomy, which should be placed as low as possible, a tracheal tube with cuff is inserted. The U-shaped incision of the skin of the neck is made according to Gluck-Soerensen, placing its base below mandible. In this way the flap, which includes skin, subcutaneous fat, superficial fascia, platisma and superficial layer of deep cervical fascia, is raised. This approach opens a wide view of the larynx and trachea and allows the exploration of the vital structures on the both sides of the neck. The median layer of the deep cervical fascia is incised immediately above the tracheostomy along the medial border of omohyoid muscle and carefully elevated up to the hyoid bone. Depending on the place and size of the laryngeal and/or the tracheal injury, the base of the flap is left cranially, on the hyoid bone, or laterally, on the omohyoid muscle. Sternohyoid muscles are resected at the insertion on the hyoid bone and the larynx is then skeletized. The endolaryngeal space is reached by midline thyrotomy or through an existing vertical fracture. After a "conservative debridement", i.e. a tissue sparing removal of damaged mucosa and cartilage, the continuity of the lacerated, but vital mucosa is provided with 4-0 or 5-0 resorptive sutures which are not under tension. Fractured parts of the cartilage which are connected to the perichondrium are returned to the anatomical position by 24 or 26 Gauge wires or by monofilament sutures. Only the parts of the cartilage completely separated from the perichondrium are removed. Major defects of the cartilage skeleton are covered with the median layer of the deep cervical fascia and connected by resorptive sutures $4-0$ to the healthy perichondrium from inside. If necessary, the median layer of the deep cervical fascia can be reinforced from outside by the undamaged sternohyoid muscle. In case of instability of laryngeal and/or tracheal ring a stent is placed endolaryngeally or endotracheally and kept there for four weeks.

Injury to the skin, subcutaneous tissue and muscles is further treated according to the principles of war wound treatment. In all cases of war injuries to the larynx and $\stackrel{\vec{A} \mathrm{G}}{\mathrm{r}}$ trachea, the wound is drained after treatment and the skm primarily closed. Postoperatively, antibiotic prophylaxis (Penicillin G 4 x 2000000 i.u. i.v. a day + Gentamicin 5 $\mathrm{mg} / \mathrm{kg}$ body weight i.v. a day in three doses of Metronidasol $3 \times 500 \mathrm{mg}$ i.v. a day) is given to 1 patients, after the recommendation of the Croatian Ministry of Health Care (14).

\section{Results}

In the postoperative course, 7 out of 8 patients w median layer of the deep cervical fascia reconstructien had no complications but the eighth died on the sixph postoperative day as a result of associated lesions of the cervical spine. During the follow-up which lasted from 5 to 18 months, no fascial necrosis was recorded. TrR patients had endolaryngeal granulations which resolved spontaneously. Following tracheostomal closure, patients had regular respiration without signs of laryng身l or tracheal stenosis. Four had good and three satisfactozy phonation. None had difficulty in swallowing.

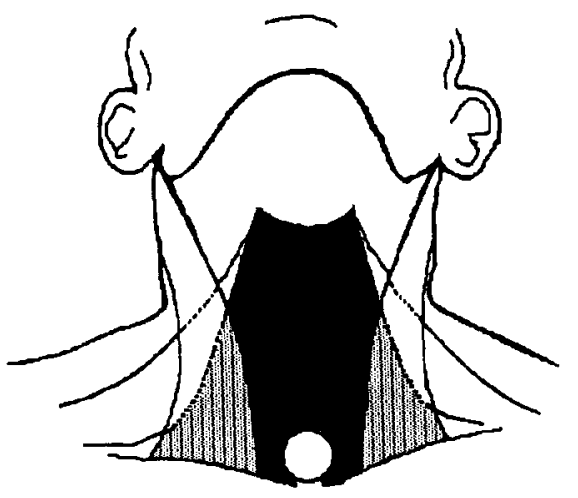

Fig 1 Median layer of deep cervical fascia; darker shaded area: the fascia between sternocleidomastoid muscles and the hyoid bone, lighter shaded area: the part of fascia under the $\frac{0}{3}$ sternocleidomastoid muscles.

\section{Discussion}

Bennett's statement in 1960 is the most pertinent to the treatment of the laryngeal trauma: "Each case presentșia different problem, and it is difficult to follow a set routine on each one. There are many other methods in which tige surgeon should be versed in order to cope with a situation, and some degree of ad-libbing is required ön each case" (15). We strictly stood by the two basic principles: surgical treatment of the lesions had to be do\&e as soon as possible within the first 24 hours after the injury and the primary procedure had to be definitive.

The type of surgical treatment will depend on the type of the weapon used, localisation and the size of the wound. Our notion that the thyroid cartilage is not solety responsible for the preservation of the laryngeal lumen after partial laryngeal resection proved itself in o 0 ar experience with war injury reconstruction. 
The shape and base of the flap, and whether it could or could not be used, was determined by the location, size, and shape of the laryngeal and tracheal wounds as well as the status of median layer of the deep cervical fascia vascularity. The flap was always approached from the sternohyoid muscle on the unharmed side of the neck with particular care to preserve its vascularization. Regardless of whether the base of the flap was cranial or lateral, it was always possible to explore the vital structures on both sides of the neck without damaging the vascularization of the flap. Due to its biological characteristics and length, the fascia also proved to be suitable for reconstruction of the first four tracheal rings, i.e. to the upper border of the formed tracheostomy.

The median layer of the deep cervical fascia proved to be useful for covering the denuded cartilaginous surfaces of the larynx and the trachea, for the formation of the lumen of the larynx and the trachea in immediate reconstruction when larger parts of cartilage skeleton were absent, and for the fixation of thyroid, cricoid or tracheal cartilage in the lesion which caused instability of laryngotracheal framework. In the latter case, a stent was placed endolaryngeally or endotracheally and the cartilage framework was fixed endo- and exolaryngeally.

The outcome of the treatment of laryngo-tracheal injuries, particularly with respect to the restoration of respiratory and phonatory functions, depended upon the following factors:

a) Early surgical exploration and immediate reconstruction.

In cases where the material for the reconstructive procedures on the larynx and the trachea is insufficient, we think the median layer of the deep cervical fascia should be used.

b) The age of the wounded.

In younger age groups cartilage framework is more elastic and less vulnerable and the wounds heal more easily.

c) The nature of the wound.

The majority of splinters from explosive devices are blunt or asymmetrically shaped and are made of steel or of a low-density material. Because of this, they quickly lose their speed in the air, do not penetrate tissues very deeply and their trace conforms to their size, remaining unchanged along the path.

\section{Conclusion}

In the treatment of exogenous laryngotracheal war injuries there is no standard surgical technique. Surgeons must be familiar with different techniques as every reconstruction of injured larynx must be approached individually. Surgical treatment of these injuries must be done during first 24 hours after the injury and the first treatment must be the definitive one. In cases in which there is not enough material for immediate reconstruction we recommend the use of median layer of the deep cervical fascia.

\section{Acknowledgements}

We thank Mr Rene Lui for translation of the paper and Drs Ana and Matko Marusic for critical review of the⿳亠口冋 manuscript.

\section{REFERENCES}

1. BENT III JP, SILvER JR, Porubsky ES. Acute laryngeal trauma: A review of 77 patients. Otolaryngoto Head Neck Surg 1993; 109: 441-49.

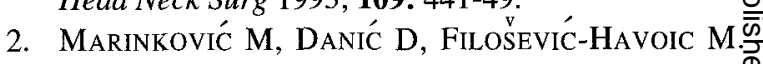
Ucesce egzogenih mehanickih povreda larinksa i traheja u ORL traumatizmu. Med Vjesn 1986; 18:197^ָ 200.

3. ZaYToun GM, Shrkhani AH, Salman SD. Head andneck injuries: 10-year experience at the American University of Beirut Medical Center. Laryngoscop 1986; 96: 899-903.

4. Jones EL, Peters AF, Gastor RM. Early manage- $\frac{\dot{1}}{A}$ ment of battle casualties in Vietnam. Arch Surgt 1986; 97: 1-15.

5. LEMAY SR. Penetrating wounds of larynx and cer cal trachea. Arch Otolaryngol 1971; 94: 558-65. 용으

6. LEOPOLD DA. Laryngeal trauma. A historical con parison of treatment methods. Arch Otolaryn 焉 1983; 109: 106-11.

7. Miller RH, DuPLeCHain JK, Penetrating wounds the neck. Otolaryngol Clin North Am 1991; 24: 29.

8. SCHAEFER SD. Acute surgical treatment of the frowtured larynx. Oper Tech Otolaryngol Head Nees Surg 1990; 1: 64-70.

9. SCHAEFER SD. The treatment of acute external laryn geal injuries. Arch Otolaryngol Head Neck Surg 1991; 117: 35-9.

10. MAISEL RH. Penetrating trauma to the neck. In Cummings $\mathrm{CW}$, editora. Otolaryngology-Head and Neck Surgery. St Louis - Philadelphia - Toronto Mosby Year Book, 1993: 1579-89.

11. KRAJINA Z. Horizontalna parcijalna laringektomija Symph Otorhinol Yug 1973; (1-2): 20-2.

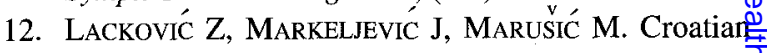
medicine in 1991 war against Croatia: A preliminary report. Croatian Med J (War Suppl.2) 1992; 33: 110 ב 19.

13. MARUŠIĆ M. Do angels cry? JAMA 1993; 270(5) 548.

14. Vodopija I, Francetić I, Tonkoví́ I, Kalení́ S LUETí V. Zastita od infekcije nakon ranjavanja Lijec Vjesn 1991; 113(7-8): 288-89.

15. Bennett T. Laryngeal trauma Laryngoscope 1960 N 70: $973-82$. 